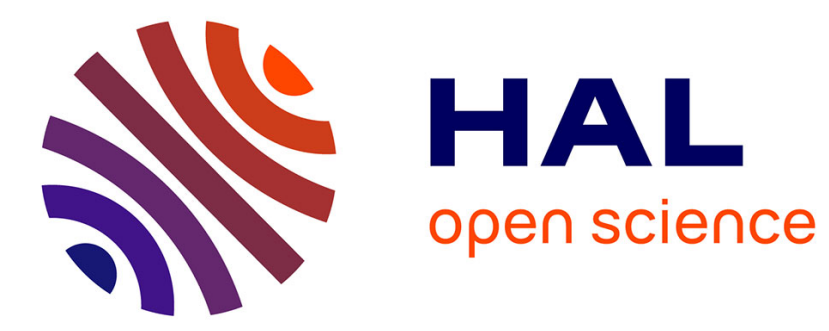

\title{
Adaptation et stratégies de coping à l'adolescence. Étude différentielle selon l'âge et le sexe
}

Florence Sordes-Ader, Sylvie Esparbès-Pistre, Pierre Tap

\section{To cite this version:}

Florence Sordes-Ader, Sylvie Esparbès-Pistre, Pierre Tap. Adaptation et stratégies de coping à l'adolescence. Étude différentielle selon l'âge et le sexe. Spirale - Revue de Recherches en Éducation , 1997, Adolescence(s), 20, pp.123-145. 10.3406/spira.1997.1596 . hal-02073771

\section{HAL Id: hal-02073771 \\ https://hal-univ-tlse2.archives-ouvertes.fr/hal-02073771}

Submitted on 20 Mar 2019

HAL is a multi-disciplinary open access archive for the deposit and dissemination of scientific research documents, whether they are published or not. The documents may come from teaching and research institutions in France or abroad, or from public or private research centers.
L'archive ouverte pluridisciplinaire HAL, est destinée au dépôt et à la diffusion de documents scientifiques de niveau recherche, publiés ou non, émanant des établissements d'enseignement et de recherche français ou étrangers, des laboratoires publics ou privés. 
Adaptation et stratégies de coping à l'adolescence. Étude différentielle selon l'âge et le sexe

Florence Sordes-Ader, Sylvie Esparbes-Pistre, Pierre Tap

\section{Citer ce document / Cite this document :}

Sordes-Ader Florence, Esparbes-Pistre Sylvie, Tap Pierre. Adaptation et stratégies de coping à l'adolescence. Étude différentielle selon l'âge et le sexe. In: Spirale. Revue de recherches en éducation, n²0, 1997. Adolescence(s) pp. 123145 ;

doi : https://doi.org/10.3406/spira.1997.1596

https://www.persee.fr/doc/spira_0994-3722_1997_num_20_1_1596

Fichier pdf généré le 31/03/2018 


\title{
Résumé
}

L'article proposé a pour but de présenter des résultats concernant les stratégies de coping à l'adolescence. Le coping est l'ensemble des modes, des comportements et des stratégies que le sujet développe pour faire face à la situation à laquelle il se trouve confronté. II est stabilisateur, permettant ainsi au sujet de maintenir une adaptation psychosociale pendant les périodes de stress. Nous avons fait passer l'échelle de coping, construite par notre équipe (ETC : échelle toulousaine de coping) à 566 adolescents, âgées de 13 à 20 ans (285 filles, 281 garçons). Cette échelle est constituée de trois champs, de quatre stratégies et de douze dimensions. Notre objectif était de savoir quelles stratégies de coping utilisaient les adolescents face à des situations stressantes. La prise en considération des différentes stratégies de coping amène à analyser la complexité des processus psychologiques et de leurs interactions. Elle nous oblige à étudier les liens entre les comportements, les compétences cognitives et les processus affectifs. Des différences sensibles apparaissent entre les garçons et les filles. Les premiers développent plus fréquemment les stratégies de contrôle et de refus, tandis que les filles utilisent de préférence le soutien social et le retrait (en particulier le repli dans l'imaginaire et l'envahissement émotionnel).

\begin{abstract}
The aim of this article is to show us coping strategies at youth. Coping is a set of modes, behaviours and strategies that the young develop for adapting himself. Coping is stabilizing, allowing to maintain psychosocial adaptation during stress period. 566 teenagers were confronted to the scale coping. This scale is composed of three fields, four strategies, and twelve dimensions. The goal is to know which coping strategies are used by teenagers when they are confronted to stress situation. It brings us to study psychologic processus and interactions. Differences appear between boys and girls. Boys develop more frequently control and denial strategies whereas girls use social support and shrinking back.
\end{abstract}




\title{
ADAPTATION ET STRATÉGIES DE COPING À L'ADOLESCENCE
}

\author{
ÉTUDE DIFFÉRENTIELLE SELON LE SEXE ET L'ÂGE
}

Résumé : L'article proposé a pour but de présenter des résultats concernant les stratégies de coping à l'adolescence. Le coping est l'ensemble des modes, des comportements et des stratégies que le sujet développe pour faire face à la situation à laquelle il se trouve confronté. Il est stabilisateur, permettant ainsi au sujet de maintenir une adaptation psychosociale pendant les périodes de stress. Nous avons fait passer l'échelle de coping, construite par notre équipe (ETC : échelle toulousaine de coping) à 566 adolescents, âgées de 13 à 20 ans (285 filles, 281 garçons). Cette échelle est constituée de trois champs, de quatre stratégies et de douze dimensions. Notre objectif était de savoir quelles stratégies de coping utilisaient les adolescents face à des situations stressantes. La prise en considération des différentes stratégies de coping amène à analyser la complexité des processus psychologiques et de leurs interactions. Elle nous oblige à étudier les liens entre les comportements, les compétences cognitives et les processus affectifs. Des différences sensibles apparaissent entre les garçons et les filles. Les premiers développent plus fréquemment les stratégies de contrôle et de refus, tandis que les filles utilisent de préférence le soutien social et le retrait (en particulier le repli dans l'imaginaire et l'envahissement émotionnel).

\section{INTRODUCTION}

A l'adolescence, le sujet supporte des tensions internes et des contraintes externes multiples. Il est inscrit dans diverses logiques aliénantes, dans des jeux d'enchaînements qui l'assujettissent. Il vit de multiples contradictions liées à l'ambiguïté des injonctions sociales, mais aussi à des difficultés de choix entre des modèles passés, des désirs actuels, des aspirations et des idéaux.

Mais, face à ces pressions, provoquant incohérence et désorganisation (mentales ou comportementales), le sujet va mettre en place une dynamique 
stratégique qui facilitera son adaptation et sa restructuration. Il se donnera alors des objectifs à atteindre, construira des projets et trouvera un itinéraire lui permettant de se réaliser, en relation avec d'autres, personnes ou groupes.

Dans ces situations, le sujet vit un stress, plus ou moins prolongé. Il y réagit de multiples façons. On appellera stratégies de coping ${ }^{1}$, l'ensemble des conduites mises en place par la personne en situation stressante, dans le but de réduire, et lorsque c'est possible d'éliminer les effets de ce stress.

Étudier les stratégies de coping, c'est analyser la dynamique des conduites émotionnelles, le stress en particulier, dans l'organisation de la vie personnelle et collective. C'est aussi se demander si une situation dramatique oblige les individus à élaborer des stratégies nouvelles, à sortir de leurs modes habituels de conduite, ou si le stress s'installe par amoncellement de tracas quotidiens, en soi et séparément non dramatiques. Étudier les réactions des adolescents en situations difficiles, c'est surtout mieux comprendre comment ils tentent de s'adapter à cette situation nouvelle et stressante.

\section{DE LA CRISE À L'ADAPTATION}

En situation critique grave, tout peut être remis en question. Lorsque tout devient négatif, le sujet se vit comme "aliéné », «assujetti ». Il ne contrôle plus rien (impuissance), plus rien n'a de sens (désignification). Il ne peut se situer par rapport à des normes (anomie), ne s'estime plus et ne cherche pas à légitimer sa vie par des valeurs, il n'a plus d'idéal (étrangeté aux valeurs), il ne croit plus en lui, ni en son devenir (absence de réalisation).

La situation de crise remet en question non seulement le style habituel du sujet, mais aussi son système identitaire (doute de soi, étonnement de ne pouvoir résoudre le problème, désancrage par rapport à sa propre histoire personnelle,...), ainsi que ses projets (obligation de réévaluer les buts et les moyens). Toute stratégie implique, par ailleurs, un triple contrôle, défini en termes de maîtrise des actes, de contrôle cognitif et de gestion des émotions.

Face à une situation difficile, le sujet refuse toute relation de stricte dépendance et désire modifier un état vécu comme un «empêchement d'être ». Devant l'insatisfaction de l'état présent, il souhaite rendre meilleur son avenir. Des changements, spontanés ou provoqués se produisent alors.

\footnotetext{
${ }^{1}$ Le terme coping du verbe anglais to cope a été diversement traduit en français (stratégies d'ajustement, d'adaptation,....). L'idée de «faire face» serait plus proche du sens initial, mais nous pouvons difficilement employer le terme de «stratégie de faire face ». Nous conserverons donc ici le terme anglais, par facilité.
} 
Ils peuvent signifier le passage d'un état à un autre, ou une succession de transformations internes aboutissant à un changement de conduite.

"Tout changement implique la transformation plus ou moins brusque et profonde d'un certain système d'équilibre, donc une phase de rupture jusqu'à l'apparition d'un nouvel équilibre. Ce processus s'accompagne d'un état de tension psychologique où se mêlent une certaine anxiété et la nostalgie d'un ordre passé, mais aussi la pression d'une urgence ou même d'une contrainte, sans oublier cependant une certaine espérance et le sel de l'aventure. » (Maisonneuve J. 1982, p. 236).

Le changement, quel qu'il soit, révèle la présence et l'interdépendance de phénomènes d'ordre cognitif, affectif et comportemental à la fois, permettant au sujet de s'adapter ou de s'ajuster au mieux, à toute situation difficile.

L'adaptation est «l'acte consistant à inventer la réaction appropriée à une situation nouvelle, appropriation des moyens adéquats à une fin donnée » (Morfaux, 1980, p. 10).

L'individu n'est «adapté » à son milieu, ne fonctionne efficacement, que dans la mesure où il parvient, lui, à construire ce milieu en fonction des conceptions qu'il s'en fait lui-même, nous dit Nuttin (1980, p. 125). Pour cet auteur, l'adaptation est un ajustement fonctionnel entre les deux pôles du processus vital : l'organisme et le milieu. Il définit l'adaptation au sens large comme «toutes les formes d'interaction qui favorisent le fonctionnement d'un organisme ou d'une personnalité », le but étant d'atteindre un état satisfaisant que l'on appelle équilibre ou état optimal d'adaptation. L'adaptation et le comportement motivé du sujet amènent à un équilibre homéostatique, (op. cit. p. 125).

Sous l'influence d'une rupture momentanée d'équilibre (due à la maladie, à un accident, etc.) entre l'organisme ou la personnalité et le milieu, rupture manifestée par un besoin, par un état de tension ou de stress intense, la conduite et sa motivation sont à concevoir comme une adaptation ou une réadaptation de l'organisme au milieu. C'est cet état de tension qui fournit la source dynamique du processus d'adaptation ainsi que le comportement luimême. Par son comportement, sa conduite, le sujet va tenter de rétablir l'équilibre «entre les deux pôles de l'interaction vitale », de transformer les situations et les choses selon sa propre conception, cherchant à réaliser ainsi, ses plans et ses projets.

Ainsi divers changements se produisent automatiquement, et viennent rompre périodiquement cet état d'équilibre entre sujet et environnement. "Cette rupture périodique produit des tensions qui ne peuvent être réduites que par un comportement adaptatif infiniment renouvelé » (op. cit. p. 130). Le sujet doit alors laisser tomber ses styles habituels et chercher, trouver, essayer ou inventer de nouvelles stratégies lui permettant une adaptation réus- 
sie. S'adapter, c'est lutter contre le stress et résoudre les problèmes posés par la situation.

\section{LE STRESS}

Le mot stress repose sur un concept flou; cause pour les uns, effet ou réponse pour les autres, il revêt différents sens selon les théories présentées, les approches physiques, physiologiques ou psychologiques engagées. Une analyse conceptuelle s'impose donc.

Le mot stress vient du latin stringere qui signifie être tendu, raide, serré. Au cours des siècles, ce terme a été employé en langue anglaise sous diverses orthographes (Cox, 1978).

Quatre conceptions apparaissent :

- La première considère le stress comme «stimulus ou agent » physique (le stress du bruit, de la chaleur, etc.) ou comme agent psychosocial ou psychologique (deuil, maladie, etc.). Le stress est subi par le sujet (Wolff, 1953, Frankenhaueuser, 1975).

- La seconde perçoit le stress non plus comme agent mais comme «réponse de l'individu », comme le résultat de l'action de l'agent (Rivolier, 1989, p. 10).

- La troisième conception définit le stress comme l'ensemble correspondant à l'agent et au résultat de l'action (Selye, 1951, 1974, 1976)

- La quatrième définition découle de la conception interactionniste, qui ne considère plus le stress comme réponse ou stimulus, mais comme l'ensemble de transactions entre le sujet et l'environnement; cette transaction surchargeant les ressources du sujet. Le stress serait le « résultat de l'interaction » entre le caractère contraignant de la situation et le sens que le sujet accorde à celle-ci. Ces travaux introduisent d'autres notions telles que la gestion des émotions, l'évaluation de la situation par le sujet, et son rapport à l'environnement, passant de modèles linéaires à un modèle transactionnel.

\subsection{Approche linéaire du stress}

Selye (1936) inspiré par les travaux de Cannon s'interroge sur la présence d'une symptomatologie stéréotypée présentée par tous les malades souffrant de différentes affections (perte d'appétit, apathie, faiblesse, expression faciale de maladie, etc.). En 1956, Selye met en évidence un «Syndrome général d'adaptation». Ce syndrome représente les modifications non spécifiques qui se produisent dans l'organisme sous l'effet d'un stress. Ces modifications constituent une réponse unique de l'organisme, quelle que soit la nature de l'agent stressant (physique, chimique ou psychique) visant à ré- 
tablir l'homéostasie. Le stress est une réponse (Selye, 1974); il s'agit d'un état de stress définit comme "la réponse non spécifique du corps à toute exigence qui s'adresse à lui ». Par «non spécifique», il entend que le même schème de réponse peut être entraîné par différents stimuli de stress ou agents de stress différents. Ce syndrome comprend trois phases :

1. La réaction d'alarme correspond à l'événement qui fait choc.

2. La phase de résistance ou d'adaptation implique la mise en place de stratégies; le sujet tente de faire face, il résiste.

3. La phase d'épuisement: le sujet s'épuise dans la mise en place de stratégies qui ne marchent pas ${ }^{2}$.

Bien que Selye demeure cantonné dans une conception biologique du stress, il développe l'idée (en 1974) qu'il faut distinguer le bon stress «eustress » (qui peut être adaptatif) et le mauvais stress «distress » (préjudiciable). Mais le stress ne peut être évité, il fait partie de la vie et apparaît même indispensable.

Il a également admis par la suite que le stimulus pouvait être psychologique, et plus particulièrement brutal, aigu (un traumatisme psychique, sorte d'équivalent psychique des agressions physiques).

Mason (physiologiste, 1975) critique l'approche de Selye. Selon lui, le profil hormonal de réponse de stress dépend de la réponse, elle-même liée à la personnalité du sujet. On passe d'un concept général (celui de Selye), à une appréhension personnalisée et spécifique ; l'individu développe des réactions selon ses caractéristiques psychiques et physiologiques, innées et acquises.

De leur côté, les psychanalystes considèrent que ce sont les constructions affectives de la première enfance qui fournissent à l'adulte sa résonance émotionnelle vis-à-vis des situations auxquelles il est confronté. Sous l'impact de situations, des pulsions enfouies et des angoisses archaïques sont réactivées.

Cependant ce modèle est restrictif et linéaire, l'individu est envisagé uniquement comme système de réactions psychiques. Le terme de stress concerne essentiellement ce que Freud désigne par «angoisse automatique », submergeant tellement les possibilités de défenses de l'individu que se crée la situation traumatique actuelle. En 1926 dans «Inhibition, symptôme et angoisse », il définit l'angoisse comme l'état qui survient quand on se trouve dans une situation dangereuse sans y être préparé.

2 Parmi les travaux concernant l'épuisement, on peut citer l'usure professionnelle ou «burnout» (Pronost et Tap, 1996). 
L'angoisse serait donc une réaction au danger à venir ou survenu. Les constructions affectives de la prime enfance sont le fondement de la résonance émotionnelle vis-à-vis des situations auxquelles le sujet est confronté. Certains psychanalystes, tel Alexander, vont insister sur l'importance de la signification que le sujet attribue à la situation.

Dans la méthode phénoménologique "le fait est mis en cause, en élargissant les limites et en s'efforçant de lui redonner sa vitalité, sa vie première » (Minkowski, 1966), c'est le « vécu » (erlebnis) qui est important (Jaspers cité par Rivolier, 1989).

Pour comprendre le "phénomène ", il ne faut pas se cantonner à l'objectivité du fait, mais prendre en considération l'expérience personnelle et subjective associée au fait.

Ainsi, différents concepts sont associés à celui de stress, le vécu personnel de l'événement, l'interaction constante entre l'organisme et l'environnement (l'ambiance) et la notion de «dynamisme » du sujet, associée à la dynamique entre le corps et l'activité mentale.

Cette prise en compte du vécu du sujet et de sa subjectivité sera centrale dans la conception interactionniste de Lazarus et Folkman (1984).

L'abandon d'un modèle mécaniste fondé sur les aspects physiologiques du stress avec la prise en compte de l'intervention des émotions et du rôle des facteurs subjectifs dans le déclenchement des réponses, amène à un schéma explicatif prenant en compte le caractère complexe de la réalité, à une conception dynamique du stress, dans l'interaction entre le sujet (sa personnalité, ses capacités, ses ressources) et l'environnement, associée à l'évaluation que le sujet peut produire. Progressivement le stress est envisagé comme un "processus multifactoriel ayant des composantes affectives, cognitives, sensorielles, viscérales, endocriniennes et comportementales en interactions les unes avec les autres »(Quintard, 1994, p. 46).

\subsection{Une conception interactionniste du stress}

Pour Lazarus (1966), l'approche du stress en tant que stimulus-réponse n'explique par pourquoi une même situation peut être considérée comme une menace par les uns, un défi par les autres, ou un fait insignifiant par d'autres encore. Personnes et environnement sont des «variables » étroitement impliquées dans des rapports interactifs.

Un stimulus peut être considéré comme «agent stressant» ou stresseur (selon la terminologie de Selye) quand il produit une réponse de stress, et la « réponse est de stress » quand elle est produite par une demande, menace, contrainte, charge ou nuisance. Le fait que l'événement soit soudain, inattendu, imprévu renforce la réaction de stress qui est alors d'autant plus intense que l'émotion engendrée est plus forte (Dantzer, 1989). 
Définir le stress c'est «mettre l'accent sur la relation entre la personne et son environnement, c'est prendre en compte les caractéristiques de la personne et la nature des événements environnants » (Lazarus et Folkman, 1984, p. 21). Le stress ne réside ni dans l'événement, ni dans l'individu, mais dans la transaction individu-environnement.

Sarafino (1990) reprenant la définition de Lazarus, conclut que "le stress est la résultante de transactions entre la personne et l'environnement, qui conduisent l'individu à percevoir une discordance, réelle ou imaginée, entre les demandes d'une situation et les ressources de ses propres systèmes biologique, psychologique et sociologique.»

Le sujet peut créer l'événement, la discordance n'étant par forcément réelle mais imaginée, représentée par le sujet comme telle, et s'il ne le crée pas, il y est de toute manière pour quelque chose, puisque c'est l'interprétation qu'il donne de la situation qui va rendre celle-ci stressante ou non pour lui.

Le stress n'est plus assimilé à un stimulus objectivement menaçant, mais à la perception que l'individu en a, dans sa relation avec les événements et les significations antérieures et actuelles, (on parle de «stress per$c ̧ u »)$. La réponse de stress est alors le résultat d'un déséquilibre entre les exigences internes ou externes et les ressources mobilisées par l'individu pour faire face à ces demandes. La situation n'est pas contraignante en ellemême, mais la contrainte naît de la signification que lui donne le sujet (évaluation cognitive). Même là où l'on peut penser qu'il y a contrainte effective (deuil, perte d'un être cher, maladie grave, attentats...), les sujets ne réagissent pas de la même façon ; l'évaluation et la signification de la situation par le sujet y est capitale. Hinckle (1973), montrant les ambiguïtés de la notion de stress, évoque la difficulté d'évaluer si un événement ou une situation est plus stressful, nocif ou pathogène, qu'un autre. Il faut toujours spécifier la population ou les individus, leurs caractéristiques, pour rendre compte de leurs réactions au changement de l'environnement: "jamais une situation sociale ou une relation interpersonnelle ne peut à elle seule être pathogène » (Paulhan et Bourgeois, 1995, p. 13).

L'évaluation va donc constituer un processus essentiel pour déterminer le degré de stress de la situation dans la relation personne-milieu. Elle tient plus grand compte des ressources perçues par le sujet, que de ses ressources réelles évaluées par d'autres.

Lazarus et Folkman (1984) distinguent deux formes d'évaluation: l'évaluation primaire et l'évaluation secondaire.

Lors de l'évaluation primaire, l'individu apprécie ce qui est en jeu dans la situation, «en quoi suis-je impliqué dans la situation?». Il peut s'agir d'une perte, d'une menace ou d'un défi. Lors de l'évaluation secon- 
daire, l'individu se demande ce qu'il peut faire pour remédier à la perte, prévenir la menace ou obtenir le bénéfice. La question est alors : «qu'est-ce que je peux faire ?». Cette évaluation oriente les stratégies de coping qui seront utilisées pour faire face à la situation stressante.

Ces processus d'évaluation sont influencés par les caractéristiques personnelles antérieures et par les variables environnementales (Paulhan, 1994, p. 107).

L'individu se distingue par sa façon d'appréhender la situation, par sa sensibilité, sa vulnérabilité, son affectivité, son interprétation des données et le style de ses réactions. Dans des conditions comparables, une personne peut réagir avec colère, une autre par la dépression ou encore par l'anxiété ou la culpabilité. D'autres personnes peuvent se sentir défiées plutôt que menacées.

Le coping entre en jeu comme réponse aux estimations du stress afin que le sujet retrouve un bien-être psychologique, un certain équilibre de vie. Pour cela, il devra mettre en œuvre diverses stratégies lui permettant de réduire l'impact de cet événement, de s'adapter à la situation, de faire face (stratégies de coping).

\section{L'APPROCHE STRATÉGIQUE}

Lorsque le sujet est confronté à une situation totalement nouvelle, qu'il entre en «turbulence», le style ne suffit pas à assurer son adaptation. $\mathrm{Ne}$ pouvant plus utiliser les processus assimilateurs ou accommodateurs, il doit prendre des risques, se faire violence, être prêt à changer, sortir de luimême, faire le deuil d'une part de lui-même. Ses stratégies, habituellement fonctionnelles, ne le sont plus forcément. Le style est remis en question.

Mages et Mendelsohn (1979), dans une étude sur le cancer, suggèrent que l'adaptation réussie peut dépendre, en partie, de l'habileté des individus à développer de nouvelles stratégies de coping et à abandonner les stratégies de coping habituelles, devenues inefficaces ou non pertinentes. La flexibilité du coping de l'individu serait un facteur-clé dans l'adaptation au cancer (Rowland, 1989).

Folkman et Lazarus (1980) ont montré le caractère dynamique du processus de coping. Celui-ci change comme peut changer l'événement stressant. Ainsi, une étude sur le stress vécu lors d'un examen, montre que la fréquence avec laquelle certaines stratégies de coping sont employées, est significativement différente d'une étape d'examen à une autre. D'après eux, le coping doit être étudié dans des contextes spécifiques. Ils proposent d'abandonner la conception évaluative globale de coping («comment faites-vous 
face? ») pour une mesure du coping dans une situation spécifique («comment faites vous face au cancer? »).

Dans ce cas, nous passons d'une psychologie catégorielle/différentielle (style) à une psychologie stratégique : le sujet doit changer d'attitudes et de comportements. Les invariants sont remis en question, ainsi que la fonction de contrôle (crise de personnalité). La situation de crise remet en question non seulement le style habituel du sujet, mais aussi son système identitaire, sa position sociale ainsi que ses projets.

Le style est fondé sur le sujet, les stratégies, elles, s'articulent dans le rapport que ce sujet entretient avec l'environnement. Cette articulation prend sens pour lui, positivement ou négativement, en termes d'objectifs à relancer, de moyens à trouver, de valorisation et de contrôle de soi à restaurer.

A vrai dire, «toute stratégie se caractérise par l'articulation, chez un acteur, individuel ou collectif, d'une logique interne finalisée et de conduites réalisatrices. Elle implique la mise en ouvre d'une énergie d'investissement et d'une dynamique de décision, dans la définition de buts (intermédiaires ou terminaux), dans le choix de moyens (matériels ou symboliques) et dans le suivi d'itinéraires facilitant l'élaboration ou l'exécution d'un projet, qui est toujours un projet de soi (même lorsque le sujet a le sentiment d'être un porteur du projet d'autrui). Cette mise en cuvre implique une régulation interactive entre les conditions externes (situations, obstacles, relations, effets d'emprise) et la dynamique de l'acteur. » (Tap et al, 1995).

\section{LE COPING COMME STRATÉGIE DE « FAIRE FACE »}

Le concept de coping s'est développé dans les années soixante, issu des théories sur le stress. A ses débuts, le terme de coping est utilisé dans deux approches distantes, celle concernant la psychologie du comportement animal, et la psychologie de l'Ego, d'inspiration psychanalytique mais mettant en avant l'importance de l'adaptation pour comprendre la dynamique du Moi.

Le modèle animal, influencé par Darwin, défend l'hypothèse selon laquelle la survie dépend de la découverte par l'animal de ce qui est prévisible et contrôlable dans son environnement, et de l'utilisation pratique de cette découverte. Le coping est alors défini comme l'ensemble des actes par lesquels l'animal contrôle les conditions environnementales aversives et diminue l'énergie d'activation (excitation). Les auteurs utilisant ce modèle, mettent en avant l'importance des comportements d'attaque-fuite (ou évitement). 
Avec le modèle de la psychologie de l'Ego, le concept de coping se modifie. Il est défini comme l'ensemble des pensées, souples et réalistes, et des actes, permettant de résoudre les problèmes surgis et de réduire le stress. Les auteurs valorisent fortement le caractère défensif des conduites du sujet, en relation avec des traumatismes ou des angoisses infantiles, et des modes habituels, inconscients.

A partir de ce modèle, Haan $(1969,1977)$ met en place un système tripartite constitué

- du coping, représentant le moi fort et fonctionnant parfaitement,

- des défenses, à partir desquelles peuvent se développer les états névrotiques,

- de la fragmentation du moi, provoquant le fonctionnement le plus désorganisé du Moi, et susceptible de se transformer en psychose.

Les auteurs ont ici tendance à minimiser l'importance de la situation, et à surévaluer les invariants personnels fondés sur des mécanismes de défense. Le coping serait ainsi vu comme un style ou comme un ensemble de « traits opérants » ou de dispositions stables de la personnalité.

Les études faites, dans ce cadre théorique, tendent aussi à sous-évaluer la complexité et la variabilité des conduites de coping, dans la mesure où elles se limitent à des références unidimensionnelles. Or, les individus, face aux multiples stress qu'ils subissent, dans leur environnement, mettent en place de multiples stratégies de coping, simultanément ou successivement.

Dans les années soixante-dix quatre-vingt, les chercheurs critiquent ces modèles réducteurs, soit parce qu'ils se centrent trop sur la situation, soit parce qu'ils se contentent de définir des traits de personnalité constituant des styles de coping. Ils vont proposer de faire plutôt appel à des modèles centrés sur les processus réels intervenant dans l'interaction entre un sujet stressé et une situation stressante.

Le coping peut être défini comme l'ensemble «des efforts cognitifs et comportementaux [par lesquels le sujet est amené à] gérer des exigences spécifiques internes et/ou externes, estimées comme mettant à l'épreuve ou excédant les ressources de la personne» (Lazarus et Folkman, 1984, p. 141). Ce processus rend compte des actes et des pensées de l'individu face à une situation inhabituelle. Il est dynamique, car il est non seulement spécifique à la situation, mais aussi au niveau de «transaction » mis en place dans la relation entre le sujet et son environnement.

Ainsi, pour dépasser le conflit, la crise ou la situation difficile qu'il vit, le sujet met en place des stratégies de coping, lui permettant de s'ajuster à la nouvelle situation ou de se défendre contre toute emprise. Le coping est 
un facteur stabilisateur permettant au sujet de maintenir une adaptation psychosociale pendant les périodes de stress.

La réponse de «faire face » va dépendre de l'expérience passée du sujet, de ses apprentissages, de ses compétences, de la mémorisation de ses échecs et de ses succès, de l'auto-évaluation, de sa capacité à répondre à la demande (Rivolier, 1989).

L'individu est un agent actif, mettant en œuvre des réponses dans sa relation avec l'environnement. Ses réponses, pour faire face à l'imprévu (ou au «trop prévu »!), vont dépendre de la signification qu'il accorde à la situation. La quantité de changement qu'un événement peut induire est à distinguer de l'impact émotionnel que cet événement peut produire. Entre les deux vient s'interposer le sens que le sujet lui accorde (Brown et Harris, 1982). Ce retentissement, de nature tout à la fois cognitive et affective, varie avec le niveau social, l'ethnie, l'âge, le sexe..., avec certaines caractéristiques individuelles, et pour finir, avec l'état émotionnel du moment.

Les représentations de l'impact événementiel varient, elles aussi, avec le niveau social, la culture et l'ethnie des sujets (Komaroff et al., 1968 ; Paykel et al., 1976 ; Janney et al., 1977 ; Askenasy et al., 1977 ; Dohrenwend et al., 1978). D'autre part, le vécu événementiel change avec l'âge (Monroe, 1982), avec le sexe ou l'état mental (Amiel-Lebigre, 1984, 1985).

L'évaluation subjective de la situation importe plus que les faits euxmêmes (Lindsay et Norman, 1980). La façon de percevoir les événements (stress perçu) est parfois plus importante que leur gravité objective. Si le stress « objectif» est considéré comme facteur déclenchant, le stress «perçu » est plutôt envisagé comme variable régulatrice ${ }^{3}$.

Face à cette situation, le sujet évalue ses propres ressources, ses capacités à la maîtriser (contrôle perçu). «Le contrôle perçu se réfère à la manière dont les individus apprécient le degré d'influence qu'ils peuvent avoir sur l'environnement » (Nuissier, 1994, p. 68). Il ne s'agit pas tant de compétences objectivées que d'évaluation subjective de capacités. En d'autres termes, le sujet a-t-il le sentiment d'avoir les compétences requises, les capacités nécessaires pour faire face à cette situation ? Le sentiment de «pouvoir-faire » est évidemment associé à la capacité d'anticiper, de prévoir. Comme le disait Paul Valéry, « ce qui est le plus vrai d'un individu et le plus lui-même, c'est son possible, que son histoire ne dégage qu'imparfai-

3 Le terme « régulateur » nous paraît plus adapté que celui de «modérateur » souvent employé dans la littérature à propos du rôle du coping par rapport au stress. Mais cette « régulation » peut en fait se traduire, on le sait par des conduites défensives, peu « contrôlées ». Le coping ne doit donc pas être réduit à un «médiateur » cognitif. Il intervient aussi bien dans la détermination même du stress (mode d'émergence) que dans ses manifestations. 
tement ». Cette évaluation porte non seulement sur ses compétences intellectuelles (impliquant le contrôle cognitif et la capacité de trouver des solutions nouvelles), mais aussi sur ses compétences affectives (contrôler ses émotions, gérer leur expression dans l'interaction sociale).

Si ses ressources ne suffisent pas, la personne peut demander de l'aide, faire appel à son réseau social. Ce ne sont pas tant les ressources sociales objectives dont les individus disposent qui peuvent moduler les effets du stress, mais plutôt la perception qu'ils en ont (disponibilité, satisfaction) et peut-être la capacité dont ils font preuve pour les rechercher et les obtenir (compétence sociale) (Bruchon-Schweitzer et Dantzer, 1994). "Les personnes faisant le mieux face à des événements stressants de la vie sont également celles qui semblent les plus attractives (populaires) auprès d'autrui » (Wortman et Dunkel-Schetter, 1987 cité in Bruchon-Schweitzer et Dantzer, 1994 p. 145). Le soutien social a un «effet tampon » sur le stress, il amortit l'impact que celui-ci peut avoir sur le sujet (Susan Gore, 1978).

Si savoir faire appel est une preuve de compétence sociale, la dépendance qu'elle peut impliquer, lorsque le sujet se repose entièrement sur une autre personne et attend que celle-ci résolve le problème à sa place, risque fort de poser problème, y compris dans la façon dont le sujet lui-même donne sens à sa dépendance.

$\mathrm{Si}$ autrui peut être soutien social dans les moments difficiles, il peut être aussi, source de stress et de tensions. Savoir s'entourer est donc une preuve de compétence sociale. Les recherches de Bettschart W. et al., (1992, pp. 421-430) sur le support social montrent qu'il aurait un effet tampon sur l'anxiété et les troubles du sommeil chez les garçons. Les filles, quant à elles, se différencieraient des garçons par un support social «ami » plus important.

\section{5. ÉTUDE EMPIRIQUE. LE COPING À L'ADOLESCENCE : DIFFÉRENCES SELON LE SEXE ET L'ÂGE}

L'objectif de cette recherche est de montrer de quelle façon les sujets font face aux situations stressantes, et si le sexe et l'âge jouent un rôle différenciateur.

De nombreuses recherches ont été réalisées sur les effets éventuels de la variable sexe. Par contre, la littérature est vague quant aux effets de l'âge sur l'utilisation des stratégies de coping. Dans ces recherches, le plus souvent, les adolescents représentent seulement un sous-échantillon d'une population plus globale (MacCrae, 1982) ou sont mêlés aux jeunes adultes, en particulier étudiants (Folkman et Lazarus, 1985). 


\subsection{Population et instrument}

Cette étude a concerné 566 adolescents (281 garçons et 285 filles), âgés de 13 à 20 ans. Ils ont été rencontrés collectivement dans leur collège ou leur lycée, aux heures de classe.

Afin de déterminer les stratégies de coping adoptées face à des situations stressantes, les adolescents ont accepté de remplir l'Échelle Toulousaine de Coping.

Partant d'une analyse critique des échelles de coping existantes, nous avons élaboré et validé une échelle (ETC) comportant quatre stratégies de coping articulées à trois champs :

- le champ comportemental, ou conatif, représente l'ensemble des processus par lesquels le sujet réagit et «se met en action » (préparation, gestion et effets de l'action);

- le champ informationnel, ou cognitif, qualifie les processus par lesquels le sujet acquiert des informations sur l'environnement, les élabore et en tient compte dans son comportement ;

- le champ émotionnel, ou affectif, est l'ensemble des processus associés aux réactions émotionnelles et à la légitimation normative des sentiments.

Après validation interne et restructuration ${ }^{4}$, cette échelle est constituée de douze dimensions, obtenues par le croisement de ces trois champs et de quatre stratégies. Ces dernières permettent au sujet de réagir aux situations stressantes, de façon différente :

a) La stratégie de contrôle $(\alpha=.79)$ fait référence au contrôle actif (Contrôle par l'action), mais aussi à l'analyse de la situation stressante, à la prise en compte d'expériences personnelles passées, en réfléchissant à des stratégies adaptées, en se donnant des objectifs, en faisant un planning (contrôle cognitif). Enfin, le contrôle émotionnel implique, de la part du sujet, de canaliser ses émotions, de ne pas paniquer.

b) La stratégie de retrait $(\alpha=.69)$ implique le repli sur soi, le retrait social, la fuite devant la situation (retrait comportemental et social), ou encore l'évitement du problème, en se réfugiant dans l'imaginaire, en recherchant

4 A l'origine, notre échelle « théorique » comprenait dix-huit dimensions, obtenues du croisement entre les trois champs et six stratégies (focalisation, soutien social, retrait, conversion, contrôle, refus). Une étude de validation interne a été effectuée sur ces mêmes 566 adolescents. Nous obtenons un coefficient de cohérence interne convenable pour l'échelle entière $(\mathrm{a}=.79)$. Cependant, concernant les stratégies, les coefficients sont plus faibles. Après ACP avec rotations varimax, nous avons fait apparaître quatre stratégies. Nous avons éliminé un item. 
de nouvelles valeurs, en développant une philosophie de la vie, ou en mettant sa confiance en Dieu (repli imaginaire et axiologique). Le sujet peut aussi s'en prendre aux autres, se défouler sur quelque chose pour libérer son angoisse, ou encore compenser par la nourriture ou par le tabac (envahissement émotionnel, addictivité).

c) La stratégie de soutien social $(\alpha=.72)$ fait référence à la participation à des activités collectives : aller chercher autrui pour se distraire, s'engager dans des activités nouvelles (coopération distractive), mais aussi partir en quête de renseignements, rechercher des conseils auprès de personnes compétentes dans le domaine concerné (soutien informationnel). Enfin, cette stratégie peut permettre de réduire le stress par la recherche de soutien émotionnel, c'est-à-dire l'aide amicale, le réconfort.

$\mathrm{d}-$ La stratégie de refus $(\alpha=.62)$ induit une résistance à l'impulsion, à l'émission de jugements irréfléchis, à une prise de recul, au choix d'activités agréables favorisant l'oubli du problème (rétention-résignation). L'individu peut aussi faire comme si le problème n'existait pas (retrait mental-dénégation), ou encore être dans l'incapacité de dire ce qu'il ressent et plaisanter sur le problème (alexithymie-humour).

\begin{tabular}{|l|l|l|l|}
\hline Stratégies & $\begin{array}{l}\text { Action } \\
\alpha=.63\end{array}$ & $\begin{array}{l}\text { Information } \\
\alpha=.62\end{array}$ & $\begin{array}{l}\text { Émotion } \\
\alpha=.45\end{array}$ \\
\hline $\begin{array}{l}\text { Contrôle } \\
\alpha=.79\end{array}$ & Contrôle par l'action & Contrôle cognitif & Contrôle émotionnel \\
\hline $\begin{array}{l}\text { Retrait } \\
\alpha=.69\end{array}$ & $\begin{array}{l}\text { Retrait comportemen- } \\
\text { tal et social }\end{array}$ & $\begin{array}{l}\text { Conversion valeurs et repli } \\
\text { dans l'imaginaire }\end{array}$ & $\begin{array}{l}\text { Envahissement émo- } \\
\text { tionnel } \\
\text { Addictivité }\end{array}$ \\
\hline $\begin{array}{l}\text { Soutien so- } \\
\text { cial } \\
\alpha=.72\end{array}$ & Coopération distractive & Soutien informationnel & Soutien émotionnel \\
\hline $\begin{array}{l}\text { Refus } \\
\alpha=.62\end{array}$ & Rétention Résignation & Retrait mental Dénégation & $\begin{array}{l}\text { Alexithymie } \\
\text { d'insensibilisation } \\
\text { Humour }\end{array}$ \\
\hline \multicolumn{2}{|c|}{ Les stratégies de coping $(\alpha=.79)$} & \\
\hline \multicolumn{2}{|c|}{} \\
\hline \multicolumn{2}{|c|}{}
\end{tabular}

Cette échelle est constituée de 53 items. Le sujet indique sur une échelle en cinq points (1 à 5) s'il réagit «pas du tout » (1) ou « très souvent » (5), à chacune des affirmations proposées.

Les analyses quantitatives sont effectuées à partir des scores obtenus par dimension, stratégie et champ.

\subsection{Résultats}

Des analyses de variance ont été réalisées en incluant l'âge et le sexe comme variables indépendantes et les quatre stratégies et leurs douze dimen- 
sions comme variables dépendantes.

Des effets significatifs concernant le sexe sont mis en évidence pour les quatre stratégies (contrôle, retrait, soutien social, refus), mais aussi pour huit dimensions sur douze (cf. tableau).

Concernant les effets de l'âge, seules les stratégies de contrôle (et ses trois dimensions) et de retrait (avec la conversion par les valeurs et l'envahissement émotionnel) sont associées à l'âge. Les deux autres stratégies, c'est-à-dire le soutien social et le refus ne sont influencées que dans une de leurs dimensions, le soutien émotionnel et le retrait mental-dénégation.

Notons qu'aucune interaction entre le sexe et l'âge n'a eu d'effet sur les stratégies. Est-ce dû à la grande variété d'âge qui annulerait les différences?

\begin{tabular}{|c|c|c|c|c|}
\hline Stratégies et dimensions & Garçons & Filles & F Fischer & $\mathrm{P}$ \\
\hline Contrôle & 52.03 & $\begin{array}{c}50.1 \\
5\end{array}$ & 5.58 & .02 \\
\hline Contrôle par l'action & 24.01 & $\begin{array}{c}22.6 \\
4 \\
\end{array}$ & 11.89 & .001 \\
\hline Retrait & 36.79 & 39.30 & 11.04 & .001 \\
\hline Conversion par les valeurs & 9.65 & 10.65 & 11.92 & .001 \\
\hline Envahissement émotionnel & 15.29 & 16.89 & 15.08 & .000 \\
\hline Soutien social & 32.27 & $\begin{array}{c}34.7 \\
3\end{array}$ & 15.51 & .000 \\
\hline Coopération distractive & 15.93 & 16.67 & 4.68 & .03 \\
\hline Soutien informationnel & 9.57 & 10.21 & 5.46 & .02 \\
\hline Soutien émotionnel & 6.77 & 7.85 & 30.60 & .000 \\
\hline Refus & 36.55 & 34.61 & 10.82 & .001 \\
\hline Rétention-résignation & 17.08 & $\begin{array}{c}16.4 \\
4 \\
\end{array}$ & 4.69 & .03 \\
\hline Alexithymie & 8.34 & 7.17 & 25.41 & .000 \\
\hline
\end{tabular}

La stratégie de contrôle

Les garçons (my $=52.03)$ utilisent davantage la stratégie de contrôle que les filles $(\mathrm{my}=50.15)(\mathrm{F}=5.58, \mathrm{p}=.02)$, en particulier le contrôle par l'action $(\mathrm{my}$ garçons $=24.01 ;$ my filles $=22.64, \mathrm{~F}=11.89, \mathrm{p}=.001$ ). Ainsi, ils font davantage face aux situations stressantes.

Ces résultats concordent avec ceux mis en évidence par d'autres auteurs. En effet, les garçons sont actifs vis à vis des situations stressantes (Seiffge-Krenke, 1994).

Le fait que les autres contrôles (émotionnel et cognitif) ne font pas l'objet de différences significatives est intéressant en soi. Même si les filles sont, comme on va le voir, plus concernées par l'envahissement émotionnel, elles s'efforcent de contrôler les tensions internes qui les habitent, aussi bien que les garçons. 
La stratégie de retrait

Les filles (my $=39.30)$ utilisent davantage la stratégie de retrait que les garçons $(\mathrm{my}=36.79)(\mathrm{F}=11.04, \mathrm{p}=.001)$. Nous constatons les mêmes résultats concernant la différence de sexe pour les dimensions de conversion par les valeurs $(\mathrm{F}=11.92, \mathrm{p}=.001)$ et pour l'envahissement émotionnel ( $\mathrm{F}$ $=15.08, \mathrm{p}=.000$ ). Ainsi, les filles changent plus que les garçons de valeurs et de comportements, et rêvent davantage (my filles $=10.65$; my garçons =9.65). Dans les situations stressantes elles se réfugient dans la passivité, dans l'imaginaire et elles rêvent à des moments meilleurs (Stone et Neale, 1984).

Elles sont aussi davantage envahies par les émotions et adoptent des conduites addictives (nourriture, fumer) (my filles $=16.89$; my garçons $=15.29$ ).

Les garçons, on l'a vu, font face par l'action, et résistent à l'envahissement des émotions. Cet état de fait peut être dû à l'apprentissage des rôles masculins et féminins. La société tolère plus facilement les manifestations émotives de la part des filles ou des femmes. Cela rejoint aussi les propos de plusieurs auteurs selon lesquels les filles réagissent aux situations problématiques par l'expression émotionnelle (Bakan 1966 ; Erikson 1968). "La vie psychique de la femme est dominée par l'affectivité.» (Deutsch 1949).

Par contre, les garçons doivent se comporter à l'image de l'homme, c'est-à-dire ne pas pleurer, ne pas montrer ses sentiments, ne pas être vulnérable. Ce statut exige donc un contrôle de la situation, des relations et des actes.

L'adolescent est dans une période de transformation induisant une certaine dépréciation. Cela pourrait donc entraîner une plus grande utilisation de la stratégie de retrait, chez la fille plus vulnérable aux émotions. Une étude récente (Nadalin 1994) a montré que si les adolescents (garçons) se contrôlent généralement mieux que les filles, c'est moins vrai chez les jeunes adultes, et c'est l'inverse à l'âge adulte, où les femmes se contrôlent mieux que les hommes (expérience de maternités, de gestion dédoublée, familiale et professionnelle, etc.) 
STRATÉGIE DE RETRAIT

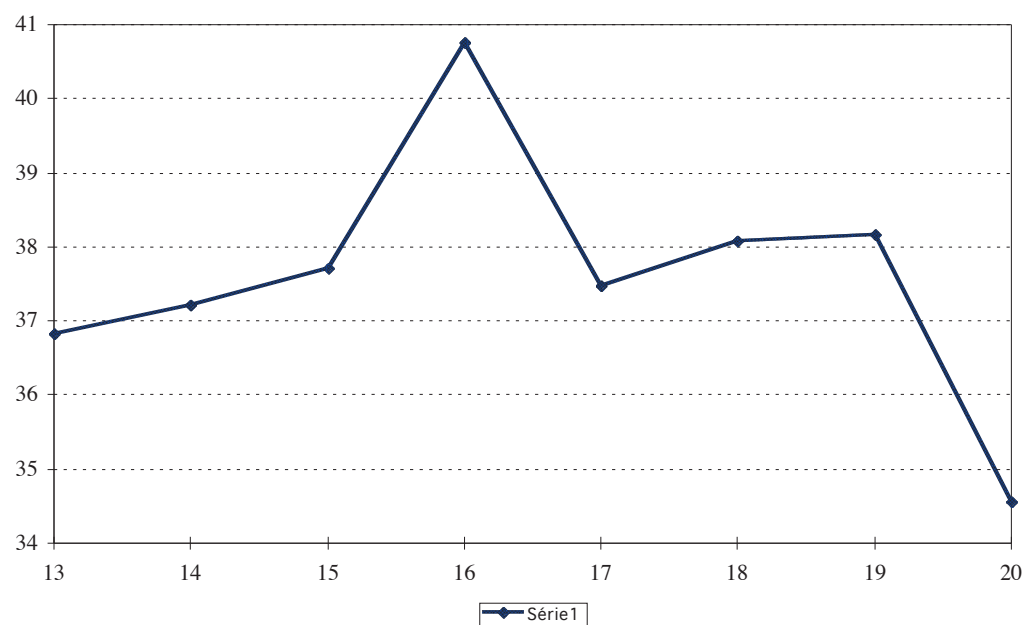

L'âge a une influence sur la stratégie de retrait $(\mathrm{F}=11.04, \mathrm{p}=.001)$, en particulier sur l'envahissement émotionnel $(\mathrm{F}=2.01, \mathrm{p}=.05)$. On constate que la stratégie de retrait et sa dimension de l'envahissement émotionnel, augmentent jusqu'à 16 ans et diminuent ensuite. Nous pouvons faire l'hypothèse selon laquelle le point culminant de la crise d'adolescence se situe à 16 ans. L'adolescent a besoin alors de s'extraire, de s'éloigner de ses parents, du contexte social,... Il se replie sur lui-même, développe des conduites addictives (tabac, alcool,...).

La stratégie de soutien social

La stratégie de soutien social est plus utilisée par les filles (my $=34.73)$ que par les garçons $(\mathrm{my}=32.27)(\mathrm{F}=15.51, \mathrm{p}=.000)$. Les mêmes résultats sont mis en évidence en faveur de filles concernant les trois dimensions liées à cette stratégie : la coopération distractive $(\mathrm{F}=4.68, \mathrm{p}=.03)$, le soutien informationnel $(\mathrm{F}=5.46, \mathrm{p}=.02)$ et le soutien émotionnel ( $\mathrm{F}$ $=30.60, \mathrm{p}=.000$ ). Elles utilisent donc plus d'aides formelles et informelles, telles que parler du problème avec d'autres personnes ( $«$ je discute du problème avec mes parents ou avec d'autres adultes »), faire partager ses émotions ( «je cherche l'aide de mes amis pour calmer mon anxiété »), chercher des activités ( $«$ je travaille en coopération avec d'autres pour me mobiliser $)$.

Ces résultats sont en accord avec de nombreuses recherches qui mettent en évidence le besoin chez les filles d'avoir un soutien social, qu'il soit 
actif, cognitif ou émotionnel (Seiffge-Krenke, 1993, 1995 ; Patterson et McCubbin, 1987). Ils sont aussi dans la continuité des travaux sur les adultes qui montrent que les femmes ont tendance à rejoindre plus facilement les réseaux sociaux que les hommes (Belle, 1981, 1987 ; Haan, 1974), mais aussi à rechercher de l'aide dans les relations extra-familiales (Ilfeld, 1980). Nous pouvons aussi dire qu'étant donné leur plus fort investissement émotionnel, elles peuvent aussi avoir davantage de soutien social.

Concernant l'effet de l'âge, nous ne mettons en évidence qu'une seule différence, concernant le soutien émotionnel $(\mathrm{F}=2.32, \mathrm{p}=.02)$. Hormis une baisse à 14 ans, il apparaît une augmentation progressive de l'utilisation de cette dimension. Ainsi, plus l'adolescent grandit, et plus il a besoin d'un soutien émotionnel. Ceci est confirmé par certaines études (Compas et al, 1993) qui remarquent que l'apprentissage des processus en relation avec les émotions continuent à progresser pendant l'adolescence, à travers les conduites amicales et amoureuses en particulier, mais sans doute aussi à l'occasion des apprentissages sportifs, et plus généralement associatifs (solidarité...)

\section{La stratégie de refus}

Les garçons $(\mathrm{my}=36.55)$ utilisent davantage la stratégie de refus que les filles $(\mathrm{my}=34.61)(\mathrm{F}=10.82, \mathrm{p}=.001)$. C'est plus particulièrement le cas pour les dimensions de rétention-résignation $(\mathrm{F}=4.69, \mathrm{p}=.001)$ et d'alexithymie-humour $(\mathrm{F}=25.41, \mathrm{p}=.000)$. Les garçons prennent donc davantage de recul (se retenir, faire le vide émotionnel...). Ceci est certainement dû au fait qu'ils traitent les problèmes lorsqu'ils se présentent. "Ils attendent et voient venir » (Seiffge-Krenke, 1994). L'alexithymie est ici à mettre en parallèle avec la négation des émotions (on ne doit pas faire voir ce que l'on ressent). Mais ces conduites de mise à distance ont aussi pour effet de les amener à nier la réalité de leur situation et de leurs émotions.

Quant à l'effet de l'âge, une seule différence émerge : le retrait mental-dénégation $(\mathrm{F}=3.68, \mathrm{p}=.001)$. Son utilisation baisse progressivement avec l'âge. Ainsi, les adolescents pratiqueraient de moins en moins la dénégation, sans doute sous l'effet des progrès perçus de leur capacité de contrôle, contrôle des émotions en particulier.

\section{CONCLUSION}

Les résultats obtenus confirment donc notre hypothèse générale selon laquelle les adolescents développent de multiples stratégies face aux situations stressantes. Sous l'effet, sans doute, de la désirabilité sociale, les stratégies de contrôle et de soutien social perçues plus positives, sont plus net- 
tement investies, aux dépens des stratégies de retrait et de refus, plus indésirables.

Des différences sensibles apparaissent entre les filles et les garçons, ici encore en relation avec des représentations et des pratiques sociales (effets liés à l'image sociale des différences de genre). Les garçons invoquent plus souvent les stratégies de contrôle et de refus, et les filles, les stratégies de soutien social et de retrait. On notera que les uns et les autres s'attribuent des stratégies perçues indésirables (retrait pour les filles ; refus pour les garçons), preuve de leur capacité à lutter contre le biais de désirabilité, au profit d'une évaluation plus objective d'eux-mêmes.

L'âge joue un rôle dans le développement de certaines stratégies. En particulier le contrôle et le besoin de soutien émotionnel augmentent avec l'âge, tandis que le retrait mental associé à la dénégation tend à diminuer entre 13 et 20 ans. Par contre, les conduites de retrait associées sans doute aux moments forts de crises personnelles, sont à leur maximum à 16 ans.

Dans le cadre de la théorie des stratégies de personnalisation, il resterait, bien sûr, à analyser l'interaction entre les stratégies de coping et les modalités d'affirmation et de continuité de soi (identité), les projets associés aux conduites de reconnaissance et d'intégration sociales. Plusieurs recherches sont actuellement engagées dans notre équipe afin de mieux comprendre ces interactions, à différents moments de la vie.

\section{Florence SORDES-ADER Sylvie ESPARBÈS-PISTRE Pierre TAP}

Université Toulouse-Le-Mirail

Abstract: The aim of this article is to show us coping strategies at youth. Coping is a set of modes, behaviours and strategies that the young develop for adapting himself. Coping is stabilizing, allowing to maintain psychosocial adaptation during stress period. 566 teenagers were confronted to the scale coping. This scale is composed of three fields, four strategies, and twelve dimensions. The goal is to know which coping strategies are used by teenagers when they are confronted to stress situation. It brings us to study psychologic processus and interactions. Differences appear between boys and girls. Boys develop more frequently control and denial strategies whereas girls use social support and shrinking back. 


\section{BIBLIOGRAPHIE}

Alexander F. (1950) La médecine psychosomatique, Payot, Paris.

Amiel-Lebigre, F., Pelc, I., \& Lagorce, A. (1984) «Événements existentiels et dépression; Une étude comparative de plusieurs types de déprimés. » Ann, Méd, Psychol, 142, 7, 937-958.

Amiel-Lebigre F. (1985) "Événements de vie et risque psychopathologique. » In : Guyotat J. \& Fédida P. Événements et psychopathologie Simep. Lyon-Villeurbanne, Paris, 9-16.

Askenasy, A.-R, Dohrenwend, B.-P, \& Dohrenwend, B.-F. (1977) «Some effects of social class abd ethnic members. Ship on judgments of the magnitude of stressful life events : research note. » Journal Health Social Behavior, 18, 432-437

Bakan, D. (1966) The duality of human existence. Chicago : Rand McNally.

Belle, D.-E. (1981) « The social network as a source of both stress and support to low income mothers. » Paper presented at the Biennial Meeting of the sociaty for Reseach in Child Development, Boston.

Belle, D.-E. (1987) « Gender differences in moderators of stress. » In : R.-D. Barnett, L. Biener et G.K. Baruch (Eds), Gender and stress. New York : The Free Press.

Bettschart, W . \& al (1992) «Événements de vie et santé psychique à la préadolescence : rôle du support social. » Neuropsychiatrie de l'Enfance, 40 (8-9), 421-430.

Brown, G.-W. \& Harris, T. (1982). «Fall-off in the reporting of life events. » Social Psychiatry. 17, 83.

Bruchon-Schweitzer, M. \& Dantzer, R. (1994) Introduction à la psychologie de la santé. PUF. Psychologie d'aujourd'hui.

Compas, B.-E., Orosan, P.-G. \& Grant, K.-E. (1993) « Adolescent stress and coping : implications for psychopathology during adolescence.» Journal of Adolescence, 16, 331-349.

Cox T. (1978) Stress, Baltimore, Maryland, University Park Press.

Dantzer R. (1989) L'illusion psychosomatique, vol 1, Odile Jacob, Paris.

Deutsch, H. (1949) La psychologie des femmes. vol. 2, trd. Benoit, Paris : PUF.

Dohrenwend, B.-S., Krasnoff, L., Askenasy, A.-R., \& Dohrenwend B.-P., (1978) «Exemplification of a method for scaling life events: The PERI life events scale. » Journal of Health and Social Behavior, 19, 205-229.

Erikson, E. (1968) «Once more the inner/space.» In : J.-H Williams (Ed), Psychology of women : selected readings. New York : Norton. 
Folkman S. \& Lazarus R.-S. (1980) «An analysis of coping in a middle aged community sample. » Journal of Health and Social Behavior, 21, 219-239.

Folkman, S. \& Lazarus, R.-S. (1985) «If it changes it must be a process : study of emotion and coping during three stages of a college examination. » Journal of Personality and Social Psychology, $48,150-170$.

Frankenhaeuser M. (1975) «Experimental approaches to the study of catecholamines and emotion.»In : Levi L. (Ed) Emotions : their parameters and measurement, New-York : Raven.

Freud S. (1926) Inhibition, symptôme et angoisse (trad. franç.) PUF (réédition 1965).

Gore, S. (1978) «The effect of social support in moderating, the health consequences of unemployment. » Journal of Health and Social Behavior, 19, 157-165.

Haan N. (1969) «A tripartite model of ego functioning : values and clinical reseach applications. » Journal of Nervous and Mental Disease, 148, 14-30.

Haan, N. (1974) « The adolescents ego model of coping and defending and comparisons with Q-sorted ideal personalities. » Genetic Psychology Monographs, 89, 273-206.

Haan N. (1977) Coping and defending : processes of self-environnment organization, New-York : Academic Press.

Hinkle L. E. Jr (1973) « The concept of « stress » in the biological and social sciences. » Science, Medecine \& Man, 1, 31-48.

Ilfield, F. W. (1980) « Coping styles of chicago adults : Description. » Journal of Human Stress, 6, 2-10.

Janney, J.-G., Masuda M. \& Holmes T. H. (1977) « Impact of a natural catastrophe one life events. » Journal Human Stress., 3, 22-34.

Komaroff, A., Masuda, M., \& Holmes, T., (1968). "The social readjustement rating scale : a comparative study of Negro, and White Americans. »J Psychosom. Res. 12, 121-128.

Lazarus R.-S. (1966) Psychological stress and the coping processes, NewYork, Mc Graw-Hill.

Lazarus R.-S. \& Folkman S. (1984) Stress, appraisal and coping, NewYork, Springer Publishing Company.

Lindsay, P.-H. \& Norman, D.-A. (1980). « Stress et émotions » In : Comportements Humains et traitements de l'information, Paris, Sciences de la vie. Roget.

MacCrae, R.-R. (1982) «Age differences in the use of coping mechanisms. » Journal of Gerontology, 37, 454-460. 
Mages, N.-L. \& Mendelson, G.-A. (1979) «Effects of cancer on patients'lives: a personological approach.» In: G.-C. Stone, F. Cohen \& N.-E. Adler (Eds), Health psychology : a handbook. San Francisco : Jossey-Bass.

Maisonneuve, J. (1982). Introduction à la psychosociologie. PUF.

Mason J.-W. (1975) « A historical view of the stress. » Journal of Human Stress, Part I et II, 1 et 2, 6-12, et 22-36.

Minkowski E. (1933) Le temps vécu, Neuchâtel, Delachaux \& Niestlé, réédition 1963.

Monroe, S. M. (1982) «Assesment of life events. Retrospective us concurrent illness. A study of 100 patients and 100 controls. » Ach. Gen. Psychiat, 39, 606-610.

Morfaux L.-M. (1980) Vocabulaire de la philosophie et des sciences humaines. Armand Colin, Paris.

Nadalin, V. (1995) Anxiété, estime de soi et stratégies de coping de l'adolescence à l'âge adulte. Étude différentielle selon le sexe et l'âge. Mémoire de maîtrise Université Toulouse le Mirail.

Nuissier, J. (1994). «Le contrôle perçu et son rôle dans les transactions entre individus et événements stressants.» (67-97) In: BruchonSchweitzer, M., et Dantzer, R. Introduction à la psychologie de la santé. PUF, Paris.

Nuttin J.-R. (1980) La structure de la personnalité, PUF, Paris, (5e édition).

Patterson, J.-M. et McCubbin, H.-I. (1987) «Adolescent coping style and behavior: conceptualization and measurement. »Journal of Adolescence, 10, 163-186.

Pauhlan I. \& Bourgeois M. (1995) Stress and coping: les stratégies d'ajustement à l'adversité, PUF, Paris.

Paykel, E.-S., Mc Guiness, B. \& Gomez J. (1976). «An Anglo-American comparison of the scaling of life events. » Br, J, Med. Psychol, 49, 237-247.

Pronost, A.M., Tap P. (1996) «La prévention du burnout et ses incidences sur les stratégies de coping. » Psychologie Française, 41-2, 165172.

Quintard B. (1994) «Du stress objectif au stress perçu.» In : M. BruchonSchweitzer \& R. Dantzer (Eds), Introduction à la psychologie de la santé, PUF, Paris, 43-66.

Rivolier J. (1989) L'homme stressé, PUF, Paris.

Rowland, J.-H. (1989) «Intrapersonal resources : coping. » In : Handbook of Psychooncology: Psychological care of the patient with cancer. Oxford University Press, New York. 
Sarafino E.P. (1990) Health psychology: biopsychosocial interactions. New-York, John Wiley \& Sons.

Seiffge-Krenke, I. (1993) «Problèmes de santé et stratégies de coping à l'adolescence. » In : P. Tap et H. Malewska-Peyre (Eds), Marginalités et troubles de la socialisation, 133-152, PUF : Paris.

Seiffge-Krenke, I. (1994) «Le coping à l'adolescence : âge, sexe et différences culturelles.»In: M. Bolognini \& al (Eds), Préadolescence : théorie, recherche et clinique. 173-183, ESF : Paris.

Seiffge-Krenke, I. (1995) Stress, coping and relationships in adolescence. LEA Publishers.

Selye H. (1936) «A syndrome produced by divers nocuous agents. » Nature, $138,2$.

Selye H. (1951) Annual report on stress, Montreal, Acta Inc.

Selye H. (1974) Stress without distress, Londres, Hodder \& Stranghton.

Selye H. (1976) «Confusion and controverses in the stress field...»J.Human Stress, 1, 37.

Stone, A.A. \& Neale, J.-M. (1984) « New measure of daily coping : developement and preliminary results. » Journal of personality and social psychology, 46, 892-906.

Tap, P., Esparbès, S. \& Sordes, F. (1995) « Stratégies de coping et personnalisation. » Sofia, Bulgarian Journal of Psychologie ; $\mathrm{n}^{\circ} 2,59-80$.

Wolff H. G. (1953) Stress and disease, Springfield, Ill., Ch. C. Thomas. 